\title{
Refractory Urticarial Vasculitis Responsive to Methotrexate: Could It Be a New Treatment Option?
}

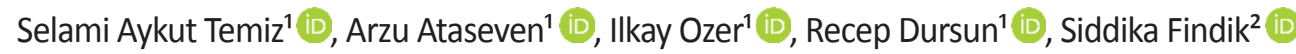 \\ ${ }^{1}$ Necmettin Erbakan University, Meram Faculty of Medicine, Department of Dermatology, Konya, Turkey \\ ${ }^{2}$ Necmettin Erbakan University, Meram Faculty of Medicine, Department of Pathology, Konya, Turkey \\ Correspondence Author: Selami Aykut Temiz \\ E-mail: aykutmd42@gmail.com \\ Received: $16.04 .2018 \quad$ Accepted: 20.07.2018
}

\begin{abstract}
Objective: Urticarial vasculitis is a chronic clinicopathologic entity characterized by clinically characterized urticarial rash, histopathologically characterized by leukocytoclastic vasculitis. The pathogenesis is thought to be developed by a type III hypersensitivity reaction similar to leukocytoclastic vasculitis.

Methods: Antihistamines and/or corticosteroids (systemic/local) may be used in patients with limited normocomplementemic urticarial vasculitis. In the literature, severe cases treated with dapsone, colchicine, hydroxychloroquine, pentoxifylline, omalizumab, mycophenolate mofetil, rituximab, cyclosporine, intravenous immunoglobulin, and cyclophosphamide have been reported in the case reports.

Results: In this case, the patient, whose symptoms had not previously responded to treatment with antihistamines, steroids, cyclosporine, and omalizumab, responded to treatment with methotrexate.

Conclusion: This case report recommends that methotrexate therapy may be a treatment option for cases of resistant urticarial vasculitis.

Keywords: Urticarial vasculitis, methotrexate, omalizumab
\end{abstract}

\section{INTRODUCTION}

Urticarial vasculitis (UV) is a chronic clinicopathologic entity characterized by clinically characterized urticarial rash, histopathologically characterized by leukocytoclastic vasculitis (1). About $5 \%$ to $10 \%$ of patients with urticarial lesions have UV. The pathogenesis is thought to be developed by a type III hypersensitivity reaction similar to leukocytoclastic vasculitis (2).

Methotrexate (MTX) is an immunosuppressive drug that has been successfully used in dermatology to treat many inflammatory diseases for almost forty years (3). In this study, the use of MTX was described for vasculitis (4).

Herein, the case of resistant urticarial vasculitis responding to MTX therapy is presented.

\section{CASE PRESENTATION}

A 31-year-old woman presented complaining of suffering from urticaria for three months. A dermatologic examination revealed common urticarial plaques on her body (Figure 1) with no angioedema. As learned from the patient's medical history, the patient's urticarial plaques lasted longer than 24 hours. There was no illness other than Hashimoto's thyroiditis in her medical history. The patient had been using $75 \mathrm{mcg}$ of Levothyroxine sodium for many years. There was no new medication use that could have triggered attacks of urticaria.

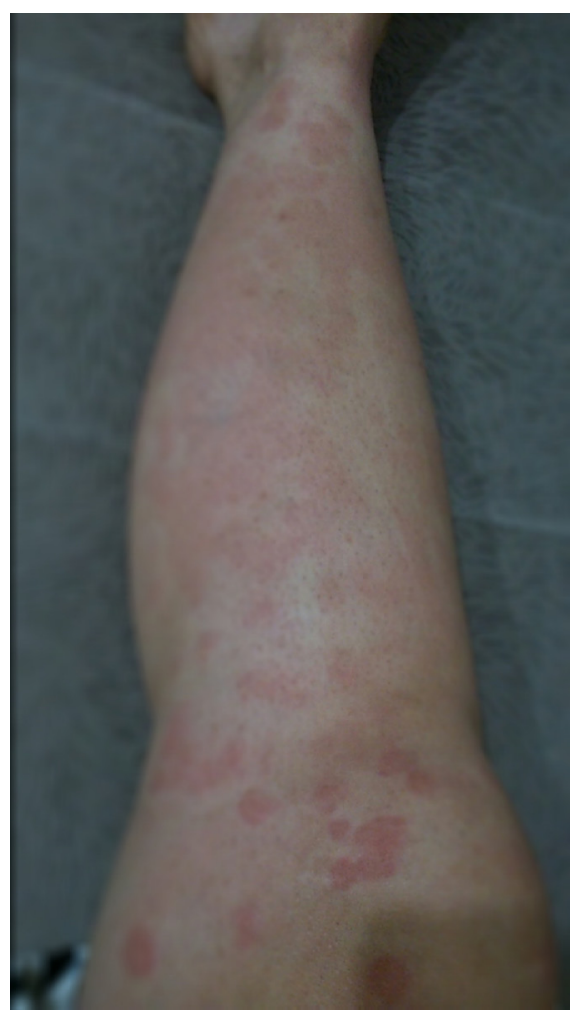

Figure 1. Common urticarial plaques on the leg. 
The patient was medicated with a Rupatadine $10 \mathrm{mg}$ tablet twice a day. After two weeks in which the symptoms did not diminish, the dosage was augmented to $10 \mathrm{mg}$ tb of Rupatadine four times a day. When the patient's symptoms were not relieved after one month of Rupatadine tablet use, a twice a day dose of Cyclosporine $100 \mathrm{mg}$ was added. After two months of an illness-free period with Cyclosporine, the urticarial attacks returned. Then, a subcutaneous treatment of Omalizumab (300 mg/28 day) was started. A $10 \mathrm{mg}$ tablet of Montelukast sodium and a $5 \mathrm{mg}$ tablet of Levocetirizine were simultaneously added to the dose. The patient's symptoms were not relieved after twelve doses of Omalizumab. Then, the Omalizumab dose was increased, and the patient received twelve doses of the Omalizumab treatment (300 mg/14 day) in six months.

A skin biopsy was taken from the patient's urticarial plaques for a differential diagnosis. Preliminary diagnoses from the biopsy were urticaria and UV. A histopathological examination showed mononuclear inflammatory infiltration in the papillary dermis with a swollen anterior fibrinoid necrosis perivascular area with eosinophils in the endothelium; as a result, it was compatible with vasculitis (Figure 2), and a direct immunofluorescence test was negative. At the laboratory review, ANA (antinuclear antibody) and other rheumatologic investigations were negative. Serum complement factors (C3, C4) levels were not found to be low.

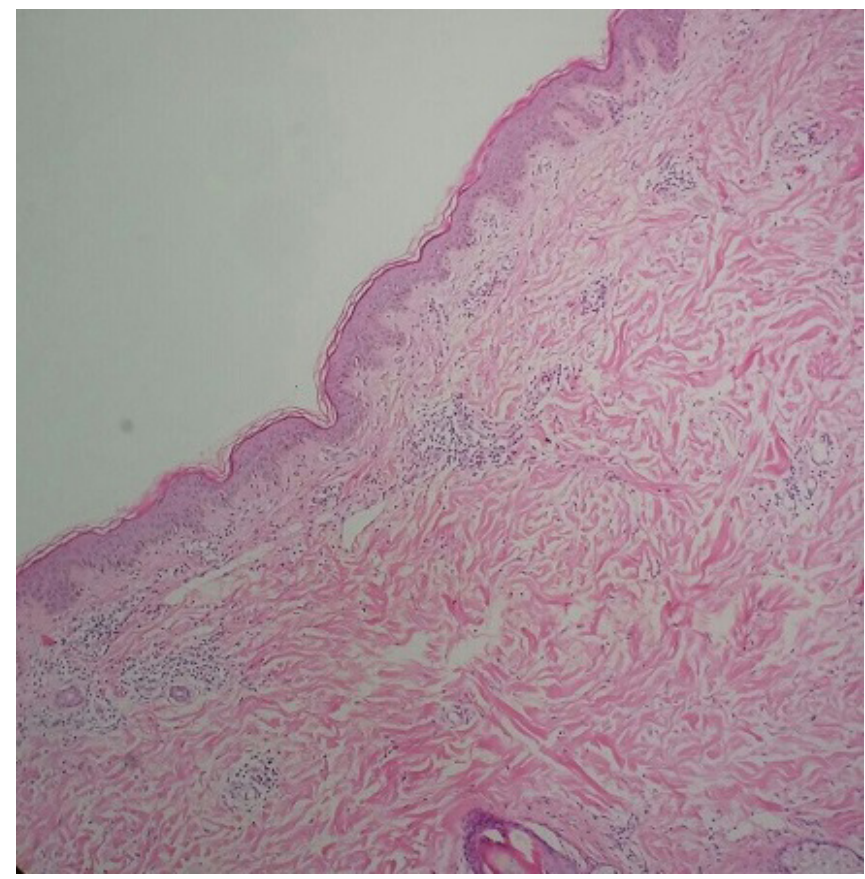

Figure 2. Mononuclear inflammatory infiltration in the papillary dermis with a swollen anterior fibrinoid necrosis, perivascular area eosinophils in endothelium, as a result it was compatible with vasculitis.

Systemic steroid therapy was added to the patient's treatment. Oral Prednisolone $48 \mathrm{mg} /$ day was started, the dose was gradually decreased, and the treatment was continued for two months. Since the patient's symptoms did not improve with systemic steroid therapy, it was decided to switch to MTX therapy. Subcutaneous MTX $10 \mathrm{mg} /$ week treatment was started, and other treatments were discontinued. The patient's complaints were retreated in the second week. The patient's symptoms have not recurred for five months after methotrexate therapy. Liver and kidney function tests were normal during methotrexate treatment. MTX therapy was discontinued at the fifth month, and there was no recurrence of the patient's symptoms at the follow-up after the fifth drug-free month. The patient provided informed consent for the publication of this case report and the included images.

\section{DISCUSSION}

UV is characterized by a cutaneous presentation resembling urticaria and inflammation of the dermal capillaries on a skin biopsy. A finding of UV recorded over long periods of 24 hours were accompanied by extreme itching, violet coloration, and purpuric foci in the center $(5,6) .20-30 \%$ of cases of UV are hypocomplemented (2). Normocomplementemic types of urticarial vasculitis are often idiopathic (7). The case was followed up clinically with an urticaria diagnosis. Then, by clinical suspicion, the case was diagnosed with normocomplemented urticarial vasculitis by biopsy and laboratory examinations.

UV treatment is planned according to the diffusiveness of skin lesions and accompanying systemic findings. Antihistamines and/or corticosteroids (systemic/local) may be used in patients with limited normocomplementemic UV. In the literature, severe cases treated with dapsone, colchicine, hydroxychloroquine, pentoxifylline, omalizumab, mycophenolate mofetil, rituximab, cyclosporine, IVIG, and cyclophosphamide have been reported in case reports $(8,9)$.

MTX has antiproliferative, immunosuppressive, and antiinflammatory effects. MTX is the most commonly used immunosuppressive agent after Prednisolone in the treatment of various skin diseases by experienced dermatologists. The fact that MTX is inexpensive, that its toxicity and side effects are well known, and that its efficacy data is easily available increases its use in dermatology (10). MTX has been used successfully in cutaneous vasculitis (11), but case reporting on use of urticarial vasculitis is limited. The MTX dose and its usage duration in the treatment of urticarial vasculitis is not clear due to the scarcity of reported cases in the literature. In the studies by Khasnis et al and Shen et al, the use of MTX as a $15 \mathrm{mg}$ of intramuscular treatment for cutaneous vasculitis is mentioned $(9,10)$, but for how many months MTX should be used is not mentioned. In this case, a patient suffering from cutaneous vasculitis was treated for five months with a subcutaneous MTX dosage of $10 \mathrm{mg} /$ week MTX and had a successful outcome. Treatment of urticarial vasculitis with methotrexate was first mentioned by Stack in the literature (12). There is another case series in which MTX was successfully used for resistant urticaria (13). 
MTX is a reliable treatment for other autoimmune diseases, and low doses seem to carry an admissible safety profile (14). The beneficial effects of methotrexate may be antiinflammatory and immunosuppressive on urticarial vasculitis (15).

\section{CONCLUSION}

The patient, whose symptoms did not respond to previous treatment with antihistamines, steroids, Cyclosporine, and Omalizumab, had a positive response to treatment with methotrexate. MTX use in UV may be suitable in selected patients. This case report recommends that MTX therapy may be a treatment option for resistant UV. The limitation of this study was that it only examined a single case. There is a need for controlled clinical trials with larger patient numbers.

Conflict of Interest: No conflict of interest was declared by the authors .

Financial Disclosure: The authors declared that this study has received no financial support.

\section{REFERENCES}

[1] Davis MDP, van der Hilst JCH. Mimickers of Urticaria: Urticarial Vasculitis and Autoinflammatory Diseases. J Allergy Clin Immunol Pract 2018;6(4):1162-70.

[2] Sjöwall C, Mandl T, Skattum L, Olsson M, Mohammad AJ. Epidemiology of hypocomplementaemic urticarial vasculitis (anti-C1q vasculitis). Rheumatology (Oxford) 2018; 57(8):140007.
[3] Kavala M, Türkoğlu Z, Özlü E. Metotreksat ve dermatolojide klinik kullanımları. Med Med J 2014; 29:104-110.

[4] Sunderkötter C, De Groot K. Therapie von Vaskulitiden und Vaskulopathien. Der Hautarzt 2008; 59:382.

[5] Fiorentino DF. Cutaneous vasculitis. J Am Acad Dermatol 2003; 48:311-40

[6] Polat AK, Belli AA, Karakus V, Dere Y. Deferasirox-induced urticarial vasculitis in a patient with myelodysplastic syndrome. An Bras Dermatol 2017; 92:59-61.

[7] Black AK. Urticarial vasculitis. Clinics in Dermatology 1999; 17:565-69.

[8] Katsambas A, Riga P. Purpura and vasculitis: unapproved treatments. Clin Dermatol 2002; 20:626-33.

[9] Khasnis A, Langford CA. Update on vasculitis. J Allergy Clin Immunol 2009; 123:1226-36.

[10] 1Shen S, O'Brien T, Yap LM, Prince HM, McCormack CJ. The use of methotrexate in dermatology: a review. Australas J Dermatol 2012; 53:1-18.

[11] Thomas-Golbanov C, Sridharan S. Novel therapies in vasculitis. Expert Opin Investig Drugs 2001; 10:1279-89.

[12] Stack PS. Methotrexate for urticarial vasculitis. Ann Allergy 1994; 72:36-38.

[13] Perez A, Woods A, Grattan CEH. Methotrexate: a useful steroidsparing agent in recalcitrant chronic urticaria. $\mathrm{Br} J$ Dermatol 2010; 162:191-94.

[14] Krause D, Schleusser B, Herborn G, Rau R. Response to methotrexate treatment is associated with reduced mortality in patients with severe rheumatoid arthritis. Arthritis Rheum 2000; 43:14-21.

[15] Cipriani P, Ruscitti P, Carubbi F, Liakouli V, Giacomelli R. Methotrexate: an old new drug in autoimmune disease. Expert Review of Clinical Immunology 2014; 10:1519-30. 